\title{
Nuevos Recursos Docentes Aplicados en Asignaturas de la Titulación de Ciencias Ambientales
}

\author{
Concepción M. Mesa-Valle ${ }^{(1)}$ y Miriam Álvarez-Corral ${ }^{(2)}$ \\ Universidad de Almería, Facultad de Ciencias Experimentales, (1) Área de Parasitología, \\ (2) Área de Química Orgánica, La Cañada de San Urbano, Almería-España \\ (e-mail: cmesa@ual.es, malvarez@ual.es)
}

Recibido Jun. 09, 2010; Aceptado Jul. 06, 2009; Versión final recibida Jul. 21, 2010

\section{Resumen}

En este trabajo se presenta el diseño por actividades de dos asignaturas con el objeto de desarrollar competencias genéricas en los estudiantes. Entre éstas se considera la expresión oral y escrita, así como competencias específicas tanto de Parasitología Ambiental, como de Química Orgánica Ambiental. También se describe la evaluación de dichas competencias. Todo esto en el marco de los objetivos y exigencias del Espacio Europeo de Educación Superior (EEES). En este contexto el EEES propone apoyar el desarrollo personal de los estudiantes para que sean competentes en sus futuras carreras profesionales y en su vida como ciudadanos activos. Este artículo expone la experiencia de varios años de trabajo con alumnos de la titulación de Ciencias Ambientales de la Universidad de Almería (España). La principal conclusión es que trabajar competencias genéricas en las asignaturas mencionadas, obliga a reducir contenidos teóricos, aunque el nuevo diseño ha permitido una mayor implicación e interés por parte del alumno, quienes adquieren competencias que antes no tenían.

Palabras clave: Ciencias Ambientales, enseñanza-aprendizaje, parasitología, química

\section{New Teaching Resources Applied to Environmental Sciences Subjects}

\begin{abstract}
In this work, the design of two specific courses by activities with the objective of developing generic competences by students is presented. Among these, oral and written communication and specific competences of Environmental Parasitology and Environmental Organic Chemistry are considered. All this has been done in the new objectives and requirements of the European Higher Education Area (EHEA). In this context, the EHEA proposes to support the personal development of the students to make them competent in their future professional careers and in their life as active citizens. This paper presents the experience of several years of work with students of Environmental Sciences at the University of Almería (Spain). The main conclusion is that working generic competences require reducing the theoretical contents of the courses, although the new design has allowed student to get more interested an involved in the courses, and to get competences that did not have before.
\end{abstract}




\section{INTRODUCCIÓN}

Desde el año 1999, en que se firmó la declaración de Bolonia, la universidad española está asistiendo a cambios profundos tanto en su organización como en las metodologías docentes, basadas ahora en el aprendizaje y no exclusivamente en la enseñanza como tradicionalmente venía ocurriendo. El estudiante pasa a ser el centro en el proceso de enseñanza-aprendizaje. Ciertos aspectos del proceso de convergencia son ya suficientemente conocidos por los distintos agentes implicados como es la organización de las titulaciones, el nuevo sistema de créditos y lo que el ECTS representa en la carga lectiva del estudiante. Sin embargo, aún hoy, hay otros puntos que para muchos siguen planteando serias dudas. La Universidad de Almería apostó desde el primer momento por este cambio y propuso a los distintos Centros la puesta en marcha de Experiencias Piloto en el EEES. La Facultad de Ciencias Experimentales asumió el reto y puso en marcha el Plan Piloto para la Titulación de Ciencias Ambientales en el curso 2005/06. A partir de este curso comienzan a funcionar grupos docentes promovidos por el Comisionado para el Espacio Europeo de Educación Superior de esta Universidad. Estos grupos docentes están formados por profesores de la Titulación pertenecientes a distintas áreas pero todos con un objetivo común: cambiar el paradigma tradicional de la Enseñanza Superior por un modelo acorde a este nuevo marco.

Este cambio en la metodología docente no sólo se está dando en España, sino que es algo mucho más extendido en el mundo y que abarca a prácticamente todas las áreas de conocimiento. De hecho, ya desde los años noventa se habla del constructivismo o aprendizaje centrado en el alumno como un cambio positivo en la docencia, como por ejemplo el estudio realizado por $\mathrm{T}$. Lord para estudiantes en laboratorios de biología (Lord, 1999), o el realizado para estudios de ingeniería (Rugarcia, 2000). La docencia basada en competencias conlleva la utilización de nuevas metodologías docentes y consecuentemente nuevos sistemas de evaluación. Es aquí donde los profesores universitarios debemos hacer una reflexión importante y tener muy presente que las actividades de aprendizaje que diseñemos para nuestros estudiantes deben ser coherentes con el sistema de evaluación. Esta adquiere una nueva dimensión al girar el aprendizaje en torno al estudiante.

Las competencias se convierten en las verdaderas protagonistas a la hora de diseñar el curriculum ya que combinan el saber, con el saber hacer y saber ser y estar de la persona. Podemos definir las competencias como la capacidad para seleccionar y movilizar conocimientos, habilidades y actitudes para responder con éxito a una determinada situación profesional. Siguiendo lo expuesto por De Miguel (De Miguel, 2006; García-Sanz, 2008) el aprendizaje basado en competencia sigue el esquema que se presenta en la figura 1. Con este trabajo queremos presentar como hemos diseñado las asignaturas de Parasitología Ambiental y Química Orgánica Ambiental en base a competencias y que recursos docentes se han empleado.

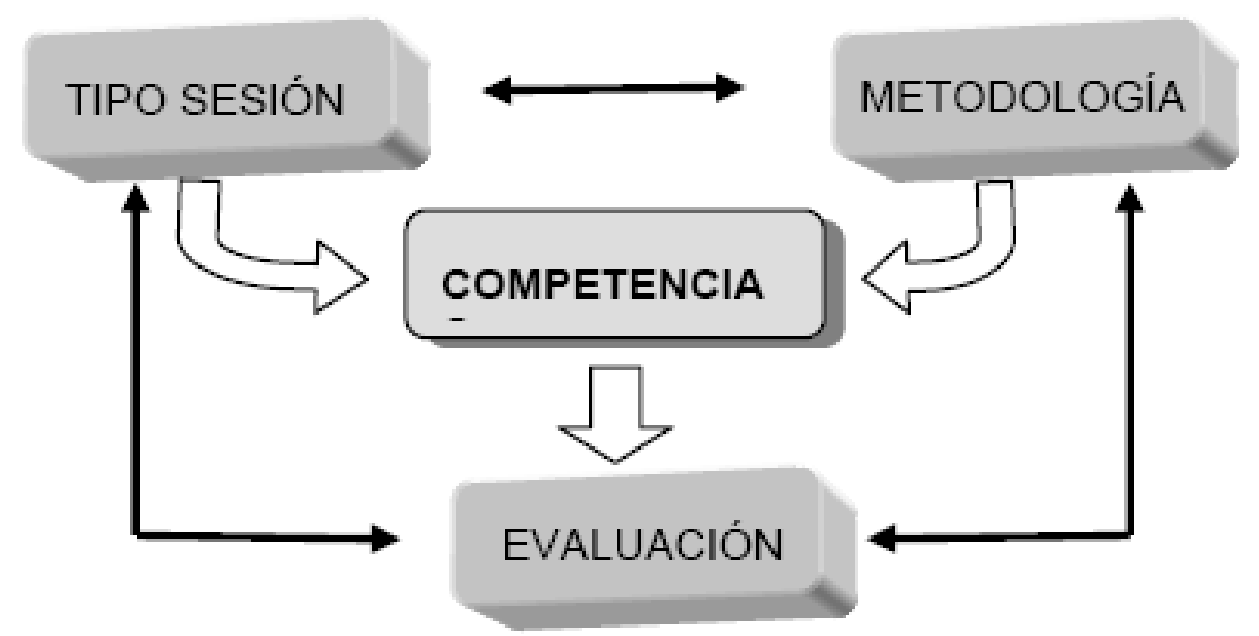

Fig. 1: Modelo basado en el desarrollo de competencias 


\section{PARASITOLOGÍA AMBIENTAL ADAPTADA AL EEES}

La asignatura de Parasitología Ambiental se trata de una asignatura optativa de primer ciclo con 4 ECTS. El primer cambio importante fue elaborar la guía docente de la asignatura. Ello me supuso la primera reflexión profunda a la hora de organizar y diseñar la docencia de la materia. Se empezaba a distinguir entre el trabajo presencial y no presencial del estudiante (4 ECTS se traduce en la UAL en 30 horas presenciales y 70 horas de trabajo autónomo por parte del alumno). Y sobre todo tenía que contextualizar perfectamente mi materia de acuerdo a las competencias específicas de la Titulación, sin olvidarme que era preciso además trabajar las competencias generales que previamente habíamos seleccionado el equipo docente de la Experiencia Piloto. Toda la información referente a la asignatura se encuentra a disposición de los alumnos en la plataforma de enseñanza virtual. Aquí están recogidas las presentaciones de cada tema así como trabajos científicos recientes relacionados con los bloques temáticos y enlaces a páginas Web que proporcionan información relacionada con la materia. También hay un calendario donde van apareciendo todas las actividades programadas y un correo electrónico que nos permite comunicarnos cualquier duda o incidencia.

Para trabajar por competencias fue preciso revisar mis propias competencias: "Deberemos mejorar nuestros conocimientos, capacidades y actitudes para lograr una mayor competencia en temas pedagógicos" (Cano-García 2008). Lo que contemplaba este nuevo escenario me llevó a dos reflexiones clave: primero, qué quiero que aprendan mis alumnos (objetivos de aprendizaje) y cómo creo que pueden aprenderlo mejor (actividades de aprendizaje); y segundo, cómo evalúo lo anterior. Es obligatorio utilizar nuevas herramientas de evaluación. La primera me obligó a determinar cuales eran los objetivos de aprendizaje de la "Parasitología Ambiental", separando el objetivo general de los objetivos específicos. Para alcanzar esos objetivos era preciso trabajar y adquirir unas competencias. Seleccioné 2 competencias generales y 2 específicas, tal y como aparece en la Tabla 1. Era la primera vez que trabajaba con estos términos y he de confesar que me fue difícil diferenciar entre "competencias" y "objetivos de aprendizaje". Los cursos de formación y la gran cantidad de material bibliográfico disponible a través de Internet me ayudaron bastante: los objetivos constituyen el camino para llegar a la adquisición de esa competencia. Las competencias mostradas en la tabla 1 , se desglosan en unas capacidades concretas tal y como se muestra en la tabla 2.

Tabla 1. Objetivos de aprendizaje y competencias de Parasitología Ambiental

\begin{tabular}{l} 
Objetivo general del curso: \\
- relacionar cambios medioambientales con la emergencia de parasitosis. \\
Objetivos específicos: \\
- definir los conceptos básicos en parasitología \\
- explicar los ciclos biológicos de los principales grupos taxonómicos de parásitos. \\
- analizar los métodos de control en parasitología \\
\hline Competencias generales \\
\hline - expresión oral y escrita \\
- búsqueda, análisis y gestión de la información \\
\hline Competencias específicas \\
\hline Conocimientos parasitológicos \\
Analizar las parasitosis mas extendidas a nivel mundial \\
\hline
\end{tabular}

Las actividades de aprendizaje para trabajar estas competencias y conseguir los objetivos de aprendizaje propuestos fueron: (1) Clases magistrales participativas: Se explican conceptos fundamentales así como los aspectos epidemiológicos directamente relacionados con el medio ambiente; y (2) Sesiones de Búsqueda de Información: Las horas presenciales del alumno con el profesor son en aula de informática, donde cada alumno dispone de un ordenador con conexión a Internet. Las clases son de 2 horas semanales de tal manera que una vez terminada la clase o clases magistrales los alumnos hacen búsquedas en bases de datos, páginas Web, etc., sobre los aspectos vistos en clase, siempre orientados por la profesora. Se ponen en común los resultados. 
Tabla 2. Capacidades a desarrollar según las competencias elegidas

\begin{tabular}{|l|l|}
\hline Competencias & Capacidades \\
\hline $\begin{array}{l}\text { Expresión oral y } \\
\text { escrita }\end{array}$ & $\begin{array}{l}\text { Organizar y presentar los contenidos o ideas que se quieran transmitir } \\
\text { siguiendo un esquema lógico. } \\
\text { Utilizar correctamente el vocabulario de la materia. } \\
\text { Presentación oral (apoyándose en recursos multimedia) y escrita de un } \\
\text { trabajo sobre un tema parasitológico relacionado con su titulación. }\end{array}$ \\
\hline $\begin{array}{l}\text { Búsqueda, análisis } \\
\text { y gestión de la } \\
\text { información }\end{array}$ & $\begin{array}{l}\text { Buscar y seleccionar información en las bases de datos relacionadas con } \\
\text { la materia. } \\
\text { Analizar y sintetizar la información obtenida }\end{array}$ \\
\hline $\begin{array}{l}\text { Conocimientos } \\
\text { parasitológicos }\end{array}$ & $\begin{array}{l}\text { Explicar el concepto de parasitismo y su relación con la ecología y la } \\
\text { evolución. } \\
\text { Identificar los grandes grupos de parásitos taxonómicamente } \\
\text { Describir los ciclos biológicos de los parásitos (protozoos, platelmintos y } \\
\text { nematelmintos). }\end{array}$ \\
\hline $\begin{array}{l}\text { Analizar las } \\
\text { parasitosis mas } \\
\text { extendidas a nivel } \\
\text { mundial }\end{array}$ & $\begin{array}{l}\text { Relacionar la epidemiología de parasitosis emergentes con los factores } \\
\text { que determinan su emergencia. } \\
\text { Evaluar los métodos de control en las parasitosis emergentes. }\end{array}$ \\
\hline
\end{tabular}

Finalmente todo el proceso era preciso evaluarlo. Como he dicho antes es el punto que me resultó más difícil y donde me surgieron muchas dudas, sobre todo a la hora de traducir los resultados en una calificación numérica. En este punto me ayudó bastante un curso de formación impartido por el Profesor Sebastián Rodríguez Espinar de la UB y miembro de la Comisión Técnica de ANECA (Rodriguez-Espinar 2000), así como numerosos documentos que hacen referencia a este punto (Benito 2005). En la actualidad todo el proceso de aprendizaje lo evalúo como sigue:

1. Examen escrito: De cada bloque temático los alumnos deben realizar un examen escrito. La media de los tres representa el 30\% de la calificación final (Si en alguno la nota no supera el 3 no se hará la media y es preciso superar un examen final de toda la materia).

2. Clases magistrales y sesiones de búsqueda de información: se valora la asistencia, participación y el interés mostrado por el alumno. El 10\% de la calificación final.

3. Elaboración de un portafolio: Aquí el alumno recoge todas las aportaciones que considere oportunas para conseguir los objetivos de aprendizaje así como las actividades propuestas por la profesora para cada bloque temático. El $60 \%$ de la calificación final.

La utilización del portafolio ha sido lo auténticamente novedoso tanto para los estudiantes como para mí. En el Seminario Internacional sobre Orientaciones Pedagógicas para la convergencia europea de educación superior (9-11 de julio de 2003) Josu Solabarrieta y Lourdes Villardón hacen referencia al uso del portafolios como técnica de evaluación en el nuevo modelo de enseñanza-aprendizaje. El uso de esta técnica de evaluación se sustenta o justifica, debido a que va acorde con la mayoría de los criterios de calidad establecidos, para un modelo innovador de aprendizaje (Barragán-Sánchez R. 2005).

Los criterios de evaluación del portafolios hacen referencia a la organización, presentación, selección de contenidos pedagógicos, desarrollo de ideas propias, habilidades para la organización y gestión etc., en definitiva estos criterios deben estar íntimamente relacionados con los objetivos y competencias seleccionadas. Se resumen en: Presentación detallada del contenido y adecuada organización de evidencias que nos permitan tener una visión integrada de todo el proceso. Redacción clara y comprensiva. Información actualizada y diversa justificando su elección. Juicio crítico. Valoración personal del proceso de enseñanza-aprendizaje señalando puntos fuertes y débiles.

Los resultados obtenidos después de aplicar todo lo anteriormente expuesto los podría resumir en los siguientes puntos: 
1. La asistencia a clase aumenta enormemente con respecto a los años en que la asignatura se impartía exclusivamente con clases magistrales.

2. El portafolio como actividad formativa despierta en los estudiantes un mayor interés por la materia y mayor implicación en el proceso de enseñanza-aprendizaje.

3. Los resultados en cuanto a la calificación numérica de los exámenes escritos son similares en ambos casos.

\section{QUÍMICA ORGÁNICA AMBIENTAL ADAPTADA AL EEES}

La asignatura Química Orgánica Ambiental es una asignatura obligatoria de tercer curso de la titulación de Ciencias Ambientales. Consta de 7 créditos ECTS, de los cuales 1.5 se dedican a prácticas de laboratorio, repartiéndose el resto entre clases magistrales, clases de problemas y seminarios de debate. Es importante señalar que los conocimientos previos que tienen los alumnos sobre química son prácticamente nulos, ya que muchos de los alumnos no eligieron esta asignatura en bachiller, e incluso los que la cursaron, vieron poco o nada de química orgánica. El alumno llega con unos prejuicios bastantes negativos y hasta un poco asustado ante algo que desconoce y que le suena a "muy difícil". Además, piensa que lo que pueda aprender no le va a servir en un futuro profesional. Todo esto ha provocado una tasa de fracaso elevada desde que se comenzó a impartir la asignatura y era necesario variar la metodología docente e intentar motivar al alumno y conseguir que adquiriese las competencias propias de la materia. Como ya se ha indicado, la titulación de Ciencias Ambientales comenzó un plan piloto de adaptación al Espacio Europeo en el curso 2005/2006, pero no ha llegado a tercero hasta el curso 2007/2008 en el que, por tanto, se aplicó el plan piloto a esta asignatura. Desde entonces se han ido introduciendo distintos recursos docentes, que se listan a continuación.

\section{Elección de competencias}

Una premisa fundamental para realizar la guía docente de la asignatura es que el centro del diseño de la asignatura son las competencias. La adquisición de competencias es la garantía del aprendizaje de los alumnos. Por tanto, la primera cuestión es seleccionar de forma correcta las competencias. Una vez marcadas éstas, las materias y actividades diseñadas tienen que ser coherentes con las mismas. Se deben centrar los objetivos de la materias, no solamente en la simple acumulación de conocimientos, sino también en las habilidades profesionales y las actitudes personales que permitan a los estudiantes ocupar el lugar correspondiente en el mundo laboral y, en general, en la sociedad. No se trataría de recibir cantidad de información sino de incidir en la calidad de la misma, esto es, capacidad para entenderla, procesarla, seleccionarla, organizarla y transformarla en conocimiento, así como la capacidad de aplicarla a diferentes situaciones y contextos sociales y/o profesionales. Las competencias genéricas se seleccionaron por el grupo docente formado por todos los profesores de las distintas asignaturas de tercer curso, como consecuencia de la mayor coordinación existente entre éstos desde que comenzaron los planes piloto. De esta forma, cada curso de la titulación selecciona unas competencias genéricas, procurando que al finalizar los estudios, el alumno haya desarrollado todas las competencias de este tipo que la Universidad de Almería ha seleccionado para sus egresados. En concreto, las elegidas para tercer curso son:

- Capacidad para resolver problemas: Capacidad para identificar, analizar, y definir los elementos significativos que constituyen un problema para resolverlo con rigor. Capacidad para aplicar los conocimientos adquiridos así como la elaboración y defensa de argumentos y la resolución de problemas de química orgánica.

- Aprendizaje de una lengua extranjera: Capacidad para realizar una lectura comprensiva de textos científicos en inglés, y poder seleccionar y resumir la información dada en esa lengua.

- Habilidad en el uso de las TIC: Capacidad para usar las fuentes de información y los medios tecnológicos para acceder a ellas; acceso y uso de bases de datos de carácter científico, búsqueda de información online. Capacidad para elaborar informes o trabajos apoyándose en el software específico de química. 
En cuanto a las competencias específicas de la asignatura, una vez definidos los contenidos de ésta, se proponen las siguientes competencias:

1) Competencias específicas relativas al conocimiento teórico (saber)

- Comprensión de los principales aspectos que configuran la estructura y propiedades de los compuestos orgánicos, en especial de aquellos con interés medioambiental.

- Comprensión de los principios fundamentales que rigen el transporte y las reacciones que sufren los compuestos orgánicos en el medioambiente.

- Conocimiento sobre aspectos relacionados con la economía de la provincia: polímeros agrícolas, plaguicidas y lucha ecológica contra plagas.

\section{2) Competencias específicas relativas al conocimiento práctico (saber hacer)}

- Capacidad para identificar las estructuras de los distintos tipos de compuestos orgánicos de preocupación medioambiental (insecticidas, herbicidas, disolventes, dioxinas, hidrocarburos aromáticos, CFCs...)

- Capacidad para relacionar los grupos funcionales en las moléculas y las propiedades físicas y químicas de dichas moléculas.

- Capacidad para realizar algunas de las técnicas habituales en un laboratorio de química orgánica.

3) Competencias específicas relativas al conocimiento ético (ser)

- Actitud crítica y reflexiva hacia los temas de química orgánica ambiental que aparecen en los medios de comunicación.

- Habilidad para informar/convencer a otras personas de la importancia de la química y su correcto uso.

\section{Selección de estrategias metodológicas}

Las competencias y objetivos guían la elección de los métodos de enseñanza, las actividades de aprendizaje de los alumnos y los sistemas de evaluación. Es necesario combinar distintos métodos para adquirir todas las competencias señaladas en el apartado anterior. A continuación se exponen los métodos que se utilizan en esta asignatura.

\section{A) Lección magistral}

Se usará la lección magistral para presentar los temas y éstos se estructurarán lógicamente para facilitar la comprensión y estudio de los contenidos. Se intentará motivar a los alumnos para que participen proponiéndole preguntas y cuestiones relacionadas con el tema. Un número adecuado de clases magistrales combinadas con seminarios sirven para trabajar las competencias relativas al conocimiento teórico. Además, el alumno tiene disponible una gran cantidad de información de la materia, tanto lecturas básicas como lecturas adicionales, en el aula virtual de la plataforma institucional de apoyo a la docencia WebCT con la que cuenta la asignatura.

\section{B) Los seminarios}

Como complemento a las clases de teoría se propondrá seminarios sobre temas de interés y actualidad. Estos seminarios serán de debate entre los propios alumnos para fomentar una actitud crítica y reflexiva hacia los temas de química orgánica ambiental (competencias específicas relativas al conocimiento ético). En concreto, se propone un seminario de debate sobre noticias de prensa relacionadas con la química orgánica. Con esta actividad se pretende motivar al alumno hacia la química, demostrando la relación real y cotidiana de nuestro entorno con esta disciplina (Álvarez-Corral, 2009)

\section{C) Resolución de ejercicios y problemas}

Se realizarán suficientes clases de problemas para que los alumnos ejerciten y pongan en práctica los conocimientos adquiridos en las clases teóricas. Son de importancia fundamental dentro del desarrollo de la asignatura, y se realizarán tanto de forma individual como colectiva, en el aula 
dentro del trabajo presencial, y fuera de ella como trabajo autónomo. Se trabajan así competencias específicas relativas al conocimiento práctico, así como la competencia genérica "resolución de problemas".

\section{D) Prácticas de laboratorio}

La formación práctica anterior se completa con cinco sesiones de laboratorio en las que el alumno aprende a utilizar algunas de las técnicas más habituales de un laboratorio de química orgánica. Para la correcta realización de las prácticas se entregará al alumno un guión de laboratorio que especifique el desarrollo de las mismas, y en el que se indicará: el objetivo perseguido con su realización; la descripción de los fundamentos teóricos en los que se basa; el material necesario para la realización; el desarrollo de la práctica, en forma de procedimiento, en el que en el que se incluya la metodología de trabajo. También vendrán bien detalladas todas las normas de seguridad necesarias para trabajar en un laboratorio de química orgánica.

Cuando un estudiante va a utilizar por primera vez una técnica, no se hace una idea de lo que se va a encontrar: material necesario, posibles errores, manejo de las muestras, dudas que le puedan surgir o simplemente el resultado que cabe esperar. Este hecho está bastante acentuado en el caso de los alumnos de Ciencias Ambientales, que tienen poco contacto con los laboratorios de química en general y de química orgánica en particular. Ante tanta expectativa están desconcentrados y se pierden en cuestiones que nada tienen que ver con la ciencia. Los alumnos aprenden más y mejor cuando visualizan los procesos antes de entrar en el laboratorio. Esto les proporciona una idea global del objetivo y de los pasos a seguir y aumenta el aprovechamiento de su tiempo de laboratorio.

Este motivo nos ha llevado a elaborar herramientas didácticas en formato de vídeo necesarias para realizar las prácticas de Química Orgánica. En concreto se han elaborado vídeo-tutoriales sobre: cómo debe construirse correctamente un montaje para calentamiento a reflujo; el seguimiento de una reacción mediante cromatografía en capa fina (CCF); y el manejo de un aparato de uso habitual en el laboratorio para retirar los disolventes volátiles de una disolución, el rotavapor. Estos vídeo-tutoriales están a disposición del alumno en el Aula Virtual de la asignatura, y además, a través del servicio de publicaciones de la Universidad de Almería, se ha editado un DVD (Álvarez-Corral, 2009).

\section{E) Aprendizaje cooperativo}

Se ha querido fomentar el trabajo en grupo como una herramienta eficaz en la resolución de tareas, de manera que sea tan importante una solución acertada, como el debate que se genera entre estudiantes. Los alumnos pueden tener más éxito que el propio profesor para hacer entender ciertos conceptos a sus compañeros. La razón fundamental de este hecho es que los compañeros están más cercanos entre sí por lo que respecta a su desarrollo cognitivo y a la experiencia en la materia de estudio (Cuseo, 1996). En concreto, las actividades planteadas son: aplicar la técnica tipo puzzle y realizar un mini-symposium con los alumnos. En concreto, la actividad planteada fue aplicar la técnica tipo puzzle para desarrollar el tema "Nomenclatura de los compuestos orgánicos", con unos resultados bastantes satisfactorios (Gisbert, 2008). En cuanto al mini-simposium, se diseña para permitir evaluar tanto la competencia genérica uso de las TICs, como una de las competencias relativas al conocimiento ético: habilidad para informar/convencer a otras personas de la importancia de la química y su correcto uso. Esta actividad se plantea al principio del cuatrimestre, para que sea un trabajo que se desarrolle durante todo el curso. Una vez creados los grupos, cada uno debe de seleccionar un tema relacionado con la Química Orgánica Ambiental que les suscite interés, puede ser de algún tipo de plaguicida, plásticos, fármaco, de toxinas como las dioxinas, etc. De ese tema, los alumnos tendrán que ir realizar un póster y defenderlo al final del curso (Mesa-Valle, 2009).

\section{CONCLUSIONES}

Trabajar competencias genéricas en las asignaturas mencionadas, obliga en cierta medida a reducir contenidos teóricos, aunque este nuevo diseño ha permitido una mayor implicación e interés por parte del alumno. Los estudiantes adquieren competencias que antes no trabajaban 
(competencias genéricas). El futuro nos dirá si realmente también serán más competentes en la materia. La utilización de nuevas herramientas de evaluación, como el portafolio, supone una mayor dedicación del profesorado, viable si los grupos de alumnos son reducidos. Todo este proceso de cambio plantea muchas incógnitas al profesorado, que debe seguir trabajando en esta línea y mejorando sus propias competencias en la enseñanza.

\section{REFERENCIAS}

Álvarez-Corral, M., Muñoz-Dorado, M., Rodríguez-García, I., Videotutoriales para el laboratorio de Química Orgánica, Universidad de Almería. ISBN: 978-84-692-6686-1 (2009)

Álvarez-Corral, M. y otros seis autores, La prensa como herramienta de aprendizaje en Química. Actas de las III Jornadas de Información sobre el EEES en la Universidad de Almería. (2009)

Barragán-Sanchez R., El Portafolio, metodología de evaluación y aprendizaje de cara al nuevo Espacio Europeo de Educación Superior. Una experiencia práctica en la Universidad de Sevilla, Revista Latinoamericana de Tecnología Educativa, 4 (1), 121-139, (2005).

Benito, A., Cruz, A., Nuevas claves para la docencia Universitaria en el Espacio Europeo de Educación Superior. Ediciones Narcea S.A. (2005)

Biggs, J., Calidad del aprendizaje universitario. Madrid: Narcea. (2004)

Cano García, M.E., La evaluación por competencias en la Educación superior. Profesorado. Revista de curriculum y formación del profesorado. 12, 3 (1-16), (2008)

Cuseo, J.B., Cooperative Learning: A Pedagogy for Addressing Contemporany Challanges \& Critical Issues in Higher Education. New Forums Press (1996)

De Miguel, M., Metodología de enseñanza y aprendizaje para el desarrollo de competencias. Orientaciones para el profesorado ante el EEES. Madrid: Alianza Editorial. (2006)

García-Sanz, M.P., Guías Docentes de Asignaturas de Grado en el EEES. Orientaciones para su elaboración. Servicio de Publicaciones, Universidad de Murcia. ISBN: 978-84-8371-762-2. (2008)

Gisbert, J., y cinco autores más., Experiences of cooperative learning and development of transferable competencies in subjects studied as part of the bachelor's degree in environmental sciences" ICERI 2008: International Conference of Education, Research and Innovation. (2008)

Lord, T., y tres autores más, Comparing Student-Centered and Teacher-Centered. Instruction in Collegue Biology Labs. The Journal of Environmental Education (1999).

Mesa-Valle, C.; Álvarez-Corral, M., Herramientas de evaluación en asignaturas de la titulación de Ciencias Ambientales en la Universidad de Almería. VI Jornadas Internacionales de Innovación Universitaria, Editorial: Universidad Europea de Madrid, ISSN/ISBN: 978-84-692-1681-1(2009)

Rodríguez-Espinar, S., La evaluación del aprendizaje de los estudiantes. Actas del I Congreso Internacional: Docencia Universitaria e Innovación. Barcelona, (2000)

Rugarcia, A., y tres autores más. The future of engineering education. A vision for a new century. Chem. ENSR. Education, 34(1), 16-25, (2000)

Solabarrieta, J., Villardon L., Concepto de evaluación en el nuevo modelo de enseñanza aprendizaje. Seminario Internacional de Orientaciones Pedagógicas para la Convergencia Europea de Educación Superior, (2003) 